\title{
Analyzing the Effects of the Ebola Virus Disease Outbreak on Maternal and Child Health Services in the Health District of Beyla, Guinea
}

\author{
Karifa Kourouma ${ }^{1,}$, , Bienvenu Salim Camara ${ }^{1}$, Delphin Kolie ${ }^{1}$, Sidikiba Sidibé $^{1,2}$, \\ Abdoul Habib Beavogui ${ }^{1}$, Alexandre Delamou ${ }^{1,2}$ \\ ${ }^{1}$ Research Unit, National Training and Research Centre in rural Health of Maferinyah, Forecariah, Guinea \\ ${ }^{2}$ Department of Public Health, Faculty of Health Sciences and Technique, University of Conakry, Conakry, Guinea \\ Email address: \\ KKourouma@maferinyah.com (K. Kourouma), bscamara@maferinyah.org (B. S. Camara), dkolie@maferinyah.org (D. Kolie), \\ layesidikiba@gmail.com (S. Sidibé), beavoguia_h@yahoo.com (A. H. Beavogui), adelamou@maferinyah.org (A. Delamou) \\ ${ }^{*}$ Corresponding author
}

\section{To cite this article:}

Karifa Kourouma, Bienvenu Salim Camara, Delphin Kolie, Sidikiba Sidibé, Abdoul Habib Beavogui, Alexandre Delamou. Analyzing the Effects of the Ebola Virus Disease Outbreak on Maternal and Child Health Services in the Health District of Beyla, Guinea. Central African Journal of Public Health. Vol. 5, No. 4, 2019, pp. 136-141. doi: 10.11648/j.cajph.20190504.11

Received: April 8, 2019; Accepted: May 29, 2019; Published: June 12, 2019

\begin{abstract}
While more efforts were needed to enhance maternal and child health services in Guinea, the country experienced the worst and longest Ebola virus disease outbreak in 2014. The aim of this study was to analyze the effects of the 2014 Ebola virus disease outbreak on antenatal care visits, institutional deliveries and vaccine coverage among children aged 0 to 11 months in the health district of Beyla in Guinea. This was an ecological study based on aggregated data from the public health facilities of the district health of Beyla, that covered three distinct periods related to the Ebola virus disease outbreak: preEbola (February $1^{\text {st }} 2013$ to January $31^{\text {st }} 2014$ ), intra-Ebola (February $1^{\text {st }} 2014$ to January $31^{\text {st }} 2015$ ) and post-Ebola (February $1^{\text {st }} 2015$ to December $\left.31^{\text {st }} 2015\right)$. Antenatal care visits, institutional deliveries and vaccination coverage of children aged 0 to 11 months significantly decreased during the intra and post-Ebola periods. The average of antenatal care visits (third visit or more) declined from $123 \%$ (pre-Ebola period) to $85 \%$ and $65 \%$ during the intra and post-Ebola periods respectively. Institutional deliveries declined from $40 \%$ to $35 \%$ then to $30 \%$ respectively ( $p<0.001$ ). Also, the average of vaccination coverage declined from $86 \%$ to $65 \%$ then to $56 \%$ respectively for BCG (p $<0.001$ ), from $90 \%$ to $50 \%$ then to $52 \%$ for Pentavalent -3 ( $\mathrm{p}<0.001$ ), and from $84 \%$ to $33 \%$ then to $48 \%$ for Oral Polio vaccine (third dose) ( $<<0.001$ ). Ebola virus disease outbreak led to persistent decrease in antenatal care visits, institutional deliveries and vaccination coverages among children aged 0 to 11 months in the district health of Beyla intra and post Ebola outbreak. Further studies, using qualitative research methods, that explore perceptions of communities and women will better guide health response during outbreak, strengthen health systems and prevent future Ebola outbreak in Guinea.
\end{abstract}

Keywords: Ebola, Antenatal Care Visits, Institutional Deliveries, Vaccination, Guinea

\section{Introduction}

Guinea is one of the countries in the world with high maternal and child mortality rates [1]. In 2012, maternal and child mortality rates in the country were 724 deaths per 100,000 live births and 67 infant deaths per 1000 live births respectively [2].

In a bid to achieve the Millennium Development Goals by 2015, efforts were made by Guinean Ministry of Health and other health stakeholders to increase women and children access to health care services between 2005 and 2012 [3]. However, despite deliberate health interventions, access to health care services remain poor for women and children in Guinea. During this period, the proportion of women who received antenatal care from qualified health care workers (HCW) increased from $82 \%$ to $85 \%$ and institutional deliveries increased from $38 \%$ to $45 \%$ [2]. Also, the proportions of vaccination coverage of children aged 0 to 11 
months for BCG (tuberculosis), the first dose of pentavalent and first dose of oral polio vaccine (OPV) were $76 \%, 82 \%$ and $85 \%$ respectively [4].

However, these achievements in maternal and child health indicators were compromised by the Ebola virus disease (EVD) outbreak in West Africa, which lasted from 2014 to 2016 and affected 3,811 people in Guinea resulting in 2,543 deaths [5]. Furthermore, health systems in affected countries were negatively impacted by the death of $\mathrm{HCW}$, restriction of health services, and decline in health facilities attendance [68]. These limitations resulted in significant reduction of health care services [9, 10]. Maternal health services, including obstetric cares (deliveries and cesarean-section), would be among the most affected health services since the risk of exposure to blood and body fluids was higher during the delivery of such services. A study in the health district of Macenta in Guinea reported a $62 \%$ drop in the number of institutional deliveries during the outbreak [11]. In addition, vaccination activities could also experience a significant decline, because during the EVD outbreak, HCW were asked to avoid direct contact and use of needles during care wherever infection control measures were poor $[12,13]$. The epidemic crisis could also interrupt the supply chain for vaccines, especially in remote areas. Delamou et al recently reported a significant decrease in the number of children vaccinated during the outbreak in the Forest Guinea region [14].

However, the effect of the EVD outbreak on maternal and child health remain poorly documented in rural Guinea. A better understanding of this effect especially in the postepidemic period would generate information to guide interventions to strengthen the health system in the postEbola period, particularly in improving maternal and child health. The aim of this study was to analyze the effects of the EVD outbreak on intra and post-epidemic coverages of antenatal care, institutional deliveries and vaccine coverage in children aged 0 to 11 months in the health district of Beyla in Guinea.

\section{Methods}

\subsection{Setting}

Guinea, located in West Africa, had more than 10 million inhabitants in 2014 [15]. Its population is predominantly rural $(67 \%)$ with an illiteracy rate of $34 \%$ [2]. The public health system is composed of 38 health districts, 30 of which were affected by the EVD outbreak.

The health district of Beyla is located in the south-eastern part of the country at $1,020 \mathrm{~km}$ from the capital city along with a population of 326000 inhabitants in 2014 [15]. The district reported 46 confirmed cases with 27 deaths (59\%), including one health care worker [16]. Its public health system is composed of a district hospital, a Communal Medical Center, 15 health centers and 29 health posts [17]. The first suspected case of EVD in the district was recorded in August 2014 and the last in January 2015 [18].

\subsection{Study Type and Period}

This was an ecological study based on an aggregated data from the public health facilities of district over three distinct periods related to the EVD outbreak: pre-Ebola (February 1rst, 2013 to January 31rst, 2014), intra-Ebola (February 1rst, 2014 to January 31 rst, 2015) and post-Ebola (February 1rst to December 31rst, 2015).

\subsection{Study Population}

All women aged 15 to 49 years who used antenatal and institutional care services and children aged from 0 to 11 months vaccinated through the extended program of immunization (EPI).

\subsection{Sources, Data Variables and Data Collection}

Data were extracted from the district health office monthly reports. For children aged from 0 - 11 months, data were collected on vaccines which include: oral polio (for poliomyelitis), pentavalent (for diphtheria, pertussis, tetanus, hepatitis B and Haemophilus influenzae type B), BCG (for tuberculosis), measles, and yellow fever.

For women aged 15 to 49 years old the following variables were considered: women who had three or more antenatal visits (ANC-3/4), institutional deliveries (normal delivery and caesarean section).

A standardized electronic Excel spreadsheet (version 2013) was prepared and used to collect information from the district health officer monthly reports. Data were collected between September 2015 and January 2016.

\subsection{Analysis and Statistics}

Data from the excel spreadsheet were analyzed using Microsoft office Excel (version 2013) and then OpenEpi (version 3.01). Results were presented using proportion (quarterly and annual trends) and the Pearson Chi-square test was used to compare the annual coverage of three distinct period. The significance level was set at $P<0.05$.

Antenatal care and institutional deliveries coverages were estimated by using the annual proportion of expected pregnancy number at national level (0.045). Vaccination coverage was calculated by using on the annual proportion of children aged from 0 - 11 months at national level (0.04) [4]. For each coverage, the annual population of the district was considered. Caesarean section rate was determined by dividing the number of caesarean by the total number of institutional deliveries (normal deliveries + caesarean).

\section{Results}

\subsection{Trends of Antenatal Care Coverage}

During the pre-Ebola period, the quarterly coverage of ANC-3/4 in the health district of Beyla decreased from a peak of $146 \%$ (February - April, 2013) to $87 \%$ (November 2013 - January, 2014) (Figure 1). 


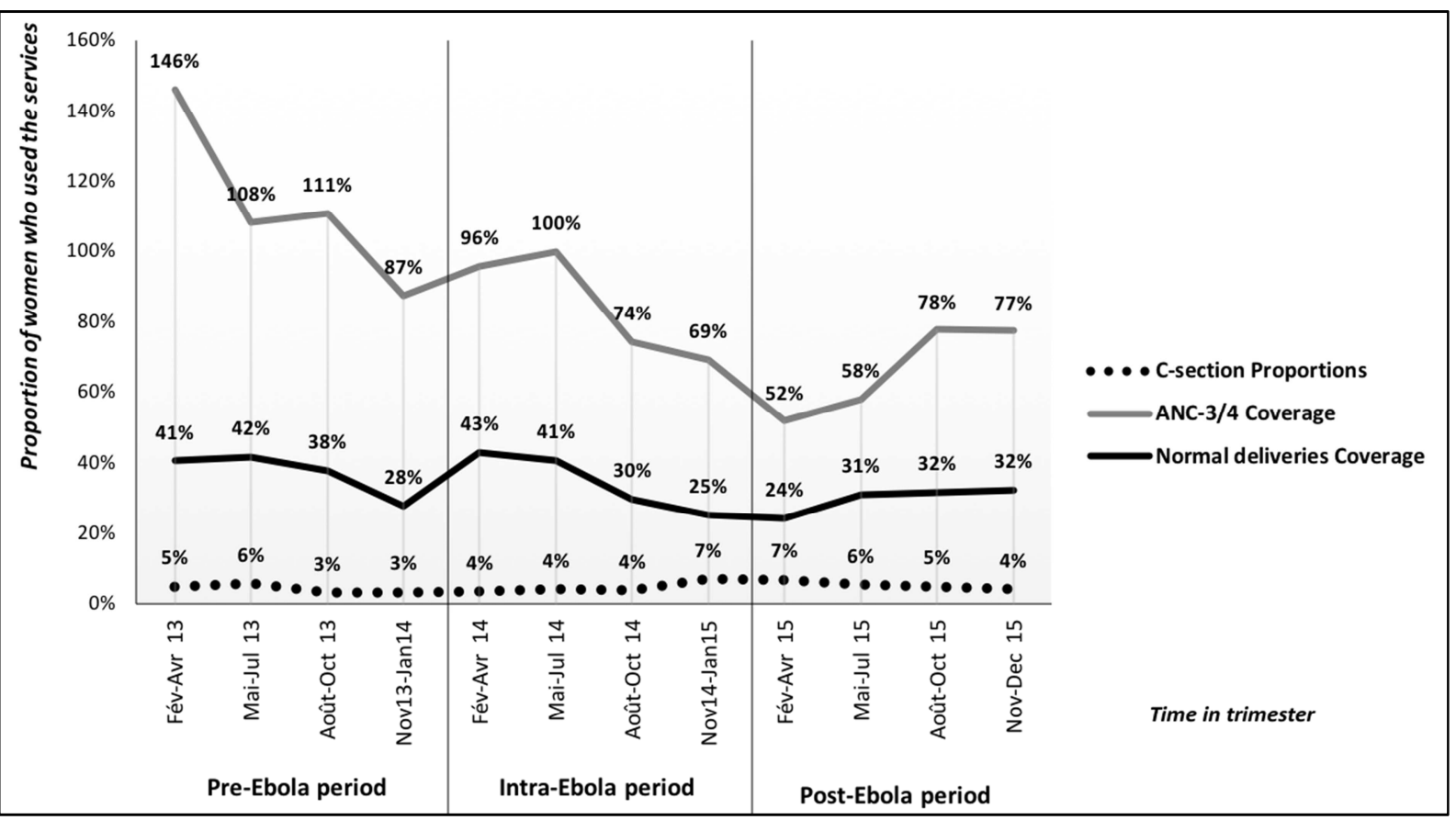

Figure 1. Quarterly trends of ANC-3/4, normal deliveries and Caesarean section coverage during the pre, intra and post-Ebola periods in the health district of Beyla in Guinea, 2013-2015.

C-section: caesarean section.

$A N C-3 / 4$ : three or more antenatal care.

During the intra-Ebola period, it decreased from a coverage of 100\% (May - July, 2014), to 69\% in the last quarter (November 2014 - January, 2015). In the post-Ebola period, the trend was on the rise from 52\% (February and April, 2015) to $77 \%$ (November and December, 2015).

The annual coverage of ANC-3/4 decreased from $123 \%$ in the pre-Ebola period to $85 \%$ and $65 \%$ during the intra-Ebola and post-Ebola period respectively. (Table 1).

Table 1. Comparison of maternal indicators coverage during pre, intra and post-Ebola periods in the health district of Beyla in Guinea (2013-2014 and 2015).

\begin{tabular}{|c|c|c|c|c|c|c|c|c|c|}
\hline \multirow[b]{3}{*}{ Indicators } & \multicolumn{9}{|l|}{ Periods } \\
\hline & Pre-Ebola & Intra-Ebola & & Intra-Ebola & Post-Ebola & & Pre-Ebola & Post-Ebola & \\
\hline & $\begin{array}{l}\text { Feb } 1^{\text {rst }}, 2013 \text { - } \\
\text { Jan } 31^{\text {rst }}, 2014\end{array}$ & $\begin{array}{l}\text { Feb } 1^{\text {rst }}, 2014 \\
- \text { Jan } 31^{\text {rst }} \\
2015 \\
\end{array}$ & $\begin{array}{l}P \text { - } \\
\text { value }\end{array}$ & $\begin{array}{l}\text { Feb } 1^{\text {rst }}, 2014 \\
- \text { Jan } 31^{\text {rst }} \\
2015\end{array}$ & $\begin{array}{l}\text { Feb } 1^{\text {rst }}- \\
\text { Dec } 31^{\text {rst }} \\
2015\end{array}$ & $\begin{array}{l}P \text { - } \\
\text { value }\end{array}$ & $\begin{array}{l}\text { Feb } 1^{\text {rst }}, 2013 \\
- \text { Jan } 31^{\text {rst }}, \\
2014 \\
\end{array}$ & $\begin{array}{l}\text { Feb } 1^{\text {rst }}- \\
\text { Dec } 31^{\text {rst }} \\
2015\end{array}$ & $P$-value \\
\hline \multicolumn{10}{|l|}{ Maternal health } \\
\hline$A N C-3 / 4^{\mathrm{a}}$ & $123 \%$ & $85 \%$ & NA & $85 \%$ & $65 \%$ & $<0.001$ & $123 \%$ & $65 \%$ & NA \\
\hline Normal deliveries & $40 \%$ & $35 \%$ & $<0.001$ & $35 \%$ & $30 \%$ & $<0.001$ & $40 \%$ & $30 \%$ & $<0.001$ \\
\hline Caesarian-Section & $5 \%$ & $4 \%$ & 0.251 & $4 \%$ & $6 \%$ & 0.003 & $5 \%$ & $6 \%$ & 0.063 \\
\hline
\end{tabular}

Legend of table.

[a]: $A N C-3 / 4$ : three or more antennal care visits.

\subsection{Trends in Institutional Deliveries Coverage}

Institutional deliveries coverage declined over the preEbola period from $41 \%$ (February - April, 2013) to $28 \%$ (November 2013 and January, 2014) (Figure 1). The trend was similar during the intra-Ebola period, declining from $43 \%$ (February - April, 2014) to $25 \%$ during the quarter

(November 2014 - January, 2015). In the post-Ebola period, the trend increased from 24\% (February - April, $2015)$ to $31 \%$ in the following quarter, then got stabilize around $32 \%$ until the end of 2015 .

The annual coverage of institutional deliveries significantly decreased from an average of $40 \%$ in the pre-
Ebola period to $35 \%$ in the intra-Ebola period then to $30 \%$ in the post-Ebola period $(\mathrm{p}<0.001)$ (Table 1).

The proportion of caesarean sections remained relatively stable over the three distinct periods, between $3 \%$ and $6 \%$ in the pre-Ebola period, and between $4 \%$ and $7 \%$ during the intra- and post-Ebola periods (Figure 1).

\subsection{Trends in Vaccination Coverage}

During the pre-Ebola period, BCG coverage increased from $67 \%$ (February - April, 2013) to a peak of $124 \%$ between the quarter (November 2013 - January 2014) (Figure 2). In the intra-Ebola period, it fell from a coverage of $99 \%$ 
in the quarter (February - April, 2014) to $37 \%$ in the quarter (November 2014 - January, 2015). After the outbreak, it increased from $39 \%$ (February and April 2015) to $79 \%$ in the following quarter, before falling to $47 \%$ during the last quarter (November - December, 2015) (Figure 2).

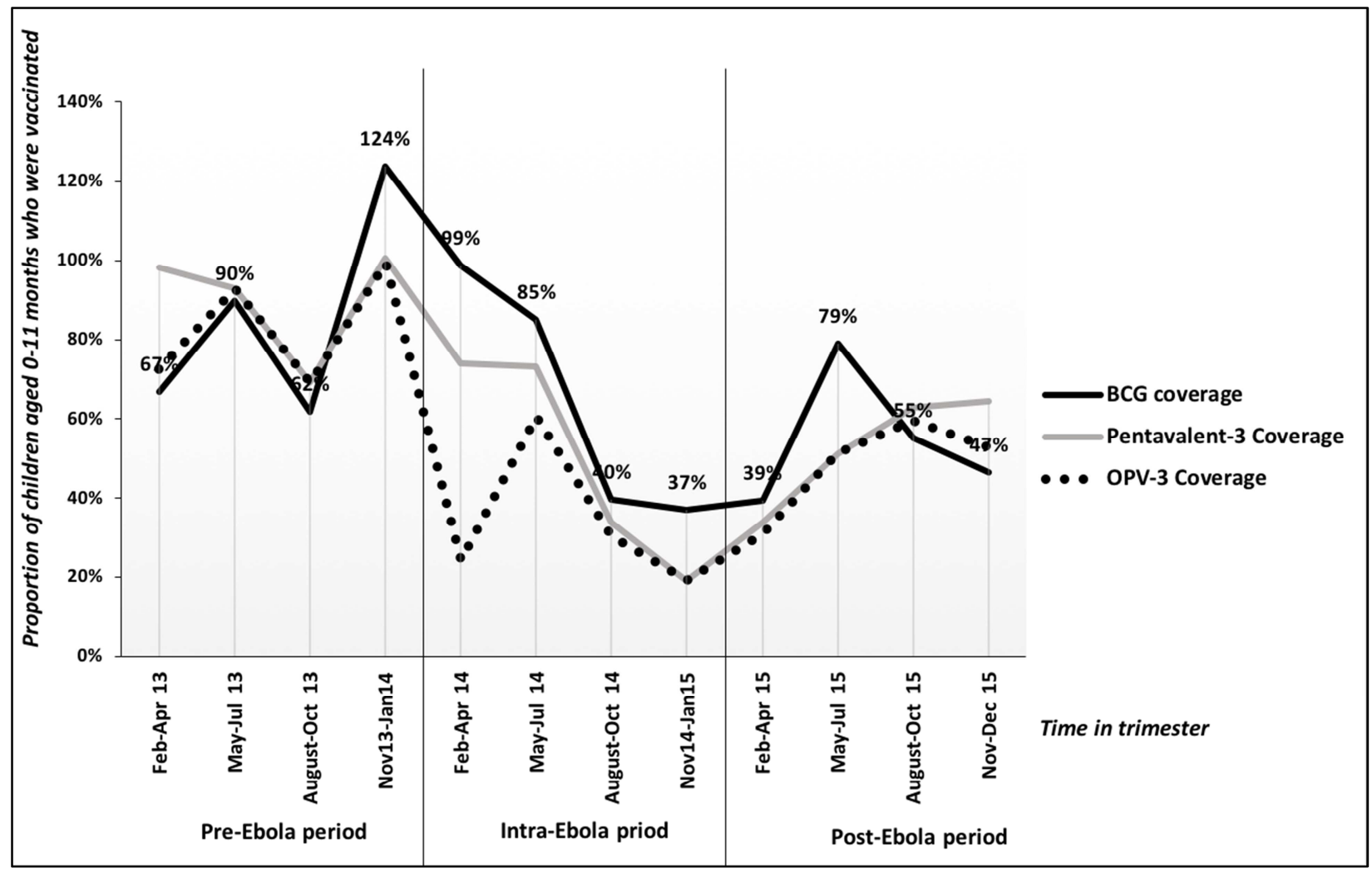

Pentavalent-3: third dose of Pentavalent vaccine; $B C G$ : Bacille Calmette-Guerin; $O P V$-3: 4th dose of polio vaccine.

Figure 2. Quarterly trends of BCG, Pentavalent-3 and OPV-3 coverage during pre, intra and post-Ebola periods in the health district of Beyla in Guinea, 2013-2015.

The average coverage of BCG significantly decreased from $86 \%$ in the pre-Ebola period to $65 \%$ in the intra period and then to $56 \%$ in the post-Ebola period $(\mathrm{p}<0.001)$ (Table 2$)$.

Table 2. Comparison of child health indicators coverage during pre, intra and post-Ebola periods in the health district of Beyla in Guinea (2013-2014 and 2015).

\begin{tabular}{|c|c|c|c|c|c|c|c|c|c|}
\hline \multicolumn{10}{|l|}{ Periods } \\
\hline & Pre-Ebola & Intra-Ebola & & Intra-Ebola & Post-Ebola & & Pre-Ebola & Post-Ebola & \\
\hline & $\begin{array}{l}\text { Feb } 1^{\text {rst }}, 2013 \\
-\operatorname{Jan}_{31} 1^{\text {rst }} \\
2014 \\
\end{array}$ & $\begin{array}{l}\text { Feb } 1^{\text {rst }}, 2014- \\
\text { Jan } 31^{\text {rst }}, 2015\end{array}$ & $P$-value & $\begin{array}{l}\text { Feb } 1^{\text {rst }}, 2014 \\
- \text { Jan } 31^{\text {rst }} \\
2015 \\
\end{array}$ & $\begin{array}{l}\text { Feb } 1^{\text {rst }}-\text { Dec } \\
31^{\text {rst }}, 2015\end{array}$ & $\begin{array}{l}P \text { - } \\
\text { value }\end{array}$ & $\begin{array}{l}\text { Feb } 1^{\text {rst }}, \\
2013 \text { - Jan } \\
31^{\text {rst }}, 2014\end{array}$ & $\begin{array}{l}\text { Feb } 1^{\text {rst }}-\text { Dec } \\
31^{\text {rst }}, 2015\end{array}$ & $P$-value \\
\hline \multicolumn{10}{|l|}{ Child health } \\
\hline Pentatavent $-3^{\mathrm{b}}$ & $90 \%$ & $50 \%$ & $<0.001$ & $50 \%$ & $52 \%$ & $<0.001$ & $90 \%$ & $52 \%$ & $<0.001$ \\
\hline
\end{tabular}

[b]: Pentavalent-3: third dose of pentavalent vaccine.

[c]: $O P V-4$ : fourth dose of polio vaccine.

NA: Not applicable.

In the pre-Ebola period, the coverage of the third dose of the pentavalent vaccine waved across quarters between $98 \%$ and $101 \%$ (Figure 2). During the intra-Ebola period, it drastically dropped from $74 \%$ in the quarter (February April, 2014) to $19 \%$ in the quarter (November 2014 January, 2015). In the post-Ebola period, the coverage had a positive trend ranging from $34 \%$ between (February and
April, 2015) to $65 \%$ (November - December, 2015). The annual coverage of the third dose of pentavalent vaccine significantly decreased from $90 \%$ in the pre-Ebola period to $50 \%$ in the intra-Ebola period and to $52 \%$ over the postEbola period $(\mathrm{p}<0.001)$ (Table 2).

During the pre-Ebola period, the coverage of the fourth dose of polio vaccine also waved between $73 \%$ in the quarter 
(February - April, 2013) and a peak of 99\% in November 2014 - January 2015 quarter (Figure 2). While the intra-Ebola period, it increased from 24\% (February and April 2014) to $61 \%$ (May - July, 2014) before falling back to its lowest coverage (19\%) over the quarter November 2014 - January 2015. During the post-Ebola period, polio vaccine coverage had a positive trend ranging from 31\% (February - April, 2015 ) to $47 \%$ (November - December, 2015). The annual coverage of the fourth dose of polio vaccine significantly decreased from $84 \%$ over the pre-Ebola period to $33 \%$ during the intra-Ebola period and to $48 \%$ the post-Ebola period ( $\mathrm{p}<$ 0.001) (Table 2).

\section{Discussion}

Our study aimed to analyze the effect of the 2014 Ebola virus disease outbreak on maternal and child health $(\mathrm{MCH})$ in rural Guinea.

The findings show a decline in antenatal care (ANC) visit coverage, institutional deliveries and vaccination of children (aged 0 to 11 months) during and even after the Ebola outbreak. Delamou et al and Camara et al also reported similar effects of Ebola outbreak on maternal and child health services in Forest Guinea, urging efforts to improve post Ebola health services $[11,14]$. The decline in health service utilization may be explained by the health workers and communities' fear of contracting Ebola virus disease and thus, restricting provision of health care services. Moreover, the fact that all the focus was on the fight against the outbreak may have also played a role [9, 19]. Thus, qualitative studies exploring community perceptions of the Ebola outbreak would contribute to better understanding of the persistence of low utilization of maternal and child health services in the post-Ebola period and to guiding recovery interventions.

Our study showed that ANC visit coverage decreased by more than half $(58 \%)$ in the post-Ebola period, compared to pre-Ebola level. This finding represents a major setback to the country's effort to increase access of pregnant women to health services, especially in rural areas where obstetric complications are common, often due to lack of follow-up during pregnancy [20]. Decrease in ANC visit coverage during the post-Ebola period could serve as an indicator to predict the risk of pregnancy complications and maternal deaths following Ebola virus disease outbreak in the health district of Beyla, which is one of the most remote districts in the country, and probably facing access to obstetric cares challenges [21].

Furthermore, half of children who benefited from vaccination before the Ebola outbreak were deprived of it during the Ebola outbreak period and more than $30 \%$ continued to lack access during the post-Ebola period. The vaccination campaigns as well as the routine activities (facility-based and outreach strategies) might have been interrupted during the outbreak because of community resistance and hostilities and/or a probable dysfunction of the vaccines supply chain.
In addition, the use of needle for some vaccines such as pentavalent could, because of fear, prevent both users and providers from receiving or administering such vaccines. The drop in the vaccination coverage among children, especially in rural areas such as Beyla has a great implication. After several years of efforts to improving child health in the country through vaccination activities, it is urgent and essential to catch-up on the vaccination of children who missed out the opportunities during the Ebola outbreak. Therefore, sustaining the routine vaccination activities regardless of any epidemic context would help to stay in line with target 4 of the Sustainable Development Goals [3].

The retrospective nature of our study was its main limitation. The Ebola outbreak context could have affected the quality of the health data reporting system, hence, affecting the quality of data we used in the study. Nevertheless, this study has the advantage of using data from all the public health facilities of the health district of Beyla over a period of three years; this allows a better comparison of the study variables.

\section{Conclusion}

This study showed the impact of the Ebola virus disease outbreak on maternal and child heath services in the health district of Bey. The results highlighted that the outbreak led to a steady decline in antenatal care coverage, institutional deliveries and vaccination of children aged 0 to 11 months. Therefore, the study calls for efforts to strengthen and maintain the health system along with maternal and child services being resilient and responsive during future outbreaks. There is also need for qualitative investigations exploring the community perceptions to help better guide and revitalize health programs in Guinea.

\section{Conflict of Interest}

All the authors do not have any possible conflicts of interest.

\section{Acknowledgements}

All our acknowledgments to the health authorities and the statistician of the Health District Office of Beyla, for facilitating the data collection.

\section{References}

[1] WHO | World Health: World Health Statistics data visualizations dashboard 2019. Monitoring health for the SDGs. [Internet] [cited 2019 May 22]; Available from: http://apps.who.int/gho/data/node.sdg.tp-1?lang=en

[2] Institut National de la Statistique, INS; Ministère du Plan et de la Coopération Internationale. Annuaire Statistique 2016. Conakry, Guinée; 2017. [Internet] [cited 2019 May 22]. Available from: http://www.statguinee.org/images/Publications/INS/annuelles/INS_annuaire_ 2016.pdf 
[3] Nations Unies. Rapport 2015. Objectifs du Millénaire pour le développement. [Internet] [cited 2017 Apr 5]. Available from: http://www.un.org/fr/millenniumgoals/reports/2015/pdf/rappo rt_2015.pdf

[4] Institut National de la Statistique INS; Ministère du Plan et de la Coopération Internationale. Enquête Démographique et de Santé et à indicateurs multiples (EDS-MICS-IV) 2012. [Internet] [cited 2019 May 22]. Available from: https://dhsprogram.com/pubs/pdf/FR280/FR280.pdf

[5] World Health Organization. Ebola Situation Report - 30 March 2016. Ebola [Internet] 2016 [cited 2017 Apr 4]. Available from: http://apps.who.int/ebola/currentsituation/ ebola-situation-report-30-march-2016.

[6] Brolin Ribacke KJ, Saulnier DD, Eriksson A, von Schreeb J. Effects of the West Africa Ebola Virus Disease on HealthCare Utilization - A Systematic Review. Front public Heal 2016; 4: 222.

[7] Ly J, Sathananthan V, Griffiths T, Kanjee Z, Kenny A, Gordon N, et al. Facility-Based delivery during the Ebola Virus Disease Epidemic in Rural Liberia: Analysis from a Cross-Sectional, Population-Based Household Survey. Kruk ME, editor. PLoS Med 2016; 13: 1002096.

[8] Elston JWT, Moosa AJ, Moses F, Walker G, Dotta N, Waldman RJ, et al. Impact of the Ebola outbreak on health systems and population health in Sierra Leone. J Public Health (Oxf) 2015; 158 .

[9] Sylla Thiam AD, Camara S, Carter J, Lama EK, Ndiaye B, Nyagero J, et al. Challenges in controlling the Ebola outbreak in two prefectures in Guinea: why did communities continue to resist? Pan Afr Med J 2015; 22.

[10] McMahon SA, Ho LS, Brown H, Miller L, Ansumana R, Kennedy CE. Healthcare providers on the frontlines: a qualitative investigation of the social and emotional impact of delivering health services during Sierra Leone's Ebola epidemic. Health Policy Plan 2016; 31: 1232-9.

[11] Camara BS, Delamou A, Diro E, Béavogui AH, El Ayadi AM, Sidibé S, et al. Effect of the 2014/2015 Ebola outbreak on reproductive health services in a rural district of Guinea: an ecological study. Trans R Soc Trop Med Hyg 2017; 1-8.
[12] Delamou A, Beavogui AH, Kondé MK, van Griensven J, De Brouwere V. Ebola: better protection needed for Guinean health-care workers. Lancet (London, England) 2015; 385: $503-4$.

[13] Centers for Disease Control and Prevention C. Recommendations for Managing and Preventing Cases of Malaria in Areas with Ebola | Ebola Hemorrhagic Fever | CDC. 2015.

[14] Delamou A, Ayadi AM El, Sidibe S, Delvaux T, Camara BS, Sandouno SD, et al. Effect of Ebola virus disease on maternal and child health services in Guinea: a retrospective observational cohort study. Lancet Glob Heal 2017; 5: 448-57.

[15] Présidence de la République, Sécrétariat Général du Gouvernement. Décret D/2015/229/PRG/SGG portant publication des résultats définitifs du troisième recensement général de la population et de l'habitation réalisé du 1er Mars au 02 Avril 2014; 2015.

[16] Coordination Nationale de lutte contre Ebola. Rapport de la Situation Epidémiologique Maladie à Virus Ebola en Guinée, 2016 (Coordination Ebola no. 702).

[17] Direction Préfectoral de la Santé (DPS) de Beyla. Rapport de la situation sanitaire de Beyla, 2015 (DPS-Beyla no. 34).

[18] Professeur René Migliani. Situation de l'épidémie de fièvre hémorragique à virus Ebola en Guinée. Bilan des données disponibles le 24 mai 2015; Available from: http://reliefweb.int/sites/reliefweb.int/files/resources/situation _epidemie_ebola_guinee_24_mai_2015.pdf

[19] Delamou A, Hammonds RM, Caluwaerts S, Utz B, Delvaux T, WHO, et al. Ebola in Africa: beyond epidemics, reproductive health in crisis. Lancet (London, England) 2014; 384: 2105.

[20] Mselle LT, Kohi TW. Healthcare access and quality of birth care: narratives of women living with obstetric fistula in rural Tanzania. Reprod Health 2016; 13: 87.

[21] Kyei-Nimakoh M, Carolan-Olah M, McCann T V. Access barriers to obstetric care at health facilities in sub-Saharan Africa—a systematic review. Syst Rev. 2017 Dec 6; 6 (1): 110. 